NASA-CR-204821

Reprinted from Journal of ATMOSPHERIC AND OCEANIC TECHNOLOGY, Vol. 10, No. 4, August 1993

American Meteorological Society

\title{
$23-434$ \\ Calibration of the Forward-scattering Spectrometer Probe: Modeling Scattering from a Multimode Laser Beam
}

\author{
EDWARD A. HOVENAC \\ Sverdrup Technology, Inc., NASA/Lewis Research Center Group, Cleveland, Ohio
}

JAMES A. LOCK

Department of Physics, Cleveland State University, Cleveland, Ohio

(Manuscript received 16 September 1992, in final form 11 January 1993)

\begin{abstract}
Scattering calculations using a more detailed model of the multimode laser beam in the forward-scattering spectrometer probe (FSSP) were carried out by using a recently developed extension to Mie scattering theory. From this model, new calibration curves for the FSSP were calculated. The difference between the old calibration curves and the new ones is small for droplet diameters less than $10 \mu \mathrm{m}$, but the difference increases to approximately $10 \%$ at diameters of $50 \mu \mathrm{m}$. When using glass beads to calibrate the FSSP, calibration errors can be minimized, by using glass beads of many different diameters, over the entire range of the FSSP. If the FSSP is calibrated using one-diameter glass beads, then the new formalism is necessary to extrapolate the calibration over the entire range.
\end{abstract}

\section{Introduction}

The forward-scattering spectrometer probe (FSSP) is an optical droplet sizing instrument frequently used to measure the diameter of cloud droplets (Dye and Baumgardner 1984). The FSSP model 100 is manufactured by Particle Measuring Systems of Boulder, Colorado, and it is calibrated at the factory to measure water droplets from 2 to $47 \mu \mathrm{m}$ in diameter (up to 95 $\mu \mathrm{m}$ for extended-range FSSPs). Microspheres such as glass beads are typically used for the calibration rather than water droplets because it is difficult to produce monodisperse water droplets of a specified size. The FSSP measurement is based on the power of light scattered by a droplet illuminated by a focused heliumneon laser beam. The scattered power is a function of droplet diameter, index of refraction, laser beam wavelength, laser beam power, and the solid angle over which the scattered light is collected. Since glass beads have an index of refraction that is different than water droplets, the scattered power is different for glass beads. When calibrating the FSSP, glass beads of one diameter are used to simulate scattering by water droplets of another diameter.

The relationship between glass-bead diameter and water-droplet diameter is calculated in three steps. First, the scattered power as a function of droplet diameter

Corresponding author address: Edward A. Hovenac, MS-77-1 Lewis Research Center, NASA, 21000 Brookpark Road. Cleveland, OH 44135 . is calculated for water droplets using Mie scattering theory. Next, the same calculation is made for glass beads. These functions will be referred to as the calibration curves. The relationship between glass-bead diameter and water-droplet diameter is determined by identifying the diameters of the water droplets that scatter the same power as a given diameter glass bead.

The calibration curves represent a model of how glass beads or water droplets scatter light in the FSSP. If a more accurate model is used to describe this process, then the calibration curves can be calculated more accurately and the droplet measurement has the potential of being more accurate as well. The purpose of this work is to recalculate the calibration curves for the FSSP using a more accurate model of the laser beam in the FSSP and to determine under what circumstances this new model is needed. This should not be confused with previous work done on the FSSP's electronic response to laser beam inhomogeneities (Baumgardner and Spowart 1990). The work by Baumgardner and Spowart represents an electronic model of the FSSP whereas this work represents an optical model.

The remainder of this paper is organized as follows. In section 2 we describe the optical properties of the FSSP and outline calibration procedures for the FSSP. In section 3 we review the calibration curve equations for the FSSP and discuss the modifications to Mie theory that enabled us to improve the model. In section 4 we show data for the new calibration curves for the FSSP and compare them with the ones traditionally used. Finally, in section 5 we comment about the sig- 
nificance of the new calibration curves and tell under what circumstances it is necessary to use them.

\section{Optical properties of the FSSP and calibration}

Before discussing calibration procedures for the FSSP it is useful to give a brief explanation of the optical system in the instrument. The optical system in the FSSP consists of the following components (refer to Fig. 1). The light source is a multimode helium-neon laser. A lens focuses the beam to a waist that is typically $200 \mu \mathrm{m}$ in diameter $(230 \mu \mathrm{m}$ for extended-range FSSPs). The waist of the beam, or the region where the beam remains focused at nearly a constant diameter, extends for a distance of roughly $2-3 \mathrm{~mm}$ along the axis of the beam. This region is the probe volume of the instrument and it is the region of the beam that droplets must cross in order to be measured by the FSSP. When a droplet crosses the laser beam in the probe volume it scatters light. A portion of the light is collected by a lens system and then is focused onto a detector. As the droplet begins to pass through the beam the scattered power rapidly increases until the droplet is fully illuminated by the beam. At some point, when the droplet is close to the center of the beam, the scattered power is at a maximum. Then as the droplet leaves the beam, the scattering decreases to zero. The FSSP makes the size determination when the scattered power is at the maximum value. If a droplet traverses a chord of the beam close to the beam's edge, then it is possible that it will never be fully illuminated by the beam and the droplet will scatter less light. This could result in a sizing error so the FSSP rejects such droplets. Details of how the FSSP rejects these droplets are given in Hovenac (1989) and are not pertinent here.

When calibrating any instrument it is usually best to use a calibration standard that is as similar as possible to the quantity that is to be measured. Since the FSSP is designed to measure water droplets, then ideally it should be calibrated with water droplets. However, this is not always practical and substitute particles are often necessary for the calibration. Glass beads, latex spheres, pinholes, and even spores have been used by various researchers for calibration of the FSSP (Hovenac and Hirleman 1991; Pinnick et al. 1981). These materials are useful because they can simulate scattering by a water droplet and they are available in a variety of specified sizes.

If monodisperse water droplets could be generated at any given size within the range of the FSSP then there would be no need to model the optical components. Calibration would consist of sending droplets of a known size through the FSSP and then adjusting the FSSP so that the output agreed with the known input. Although droplet generators can produce very repeatable, highly monodisperse droplets, they usually produce these droplets at only a few specific diameters, over a limited range, and with a great deal of operator difficulty.

Since detailed calibration using droplets is not practical, a limited calibration making use of a droplet generator and the water droplet calibration curve is possible. This calibration is performed by passing a stream of droplets of a known size through the probe volume

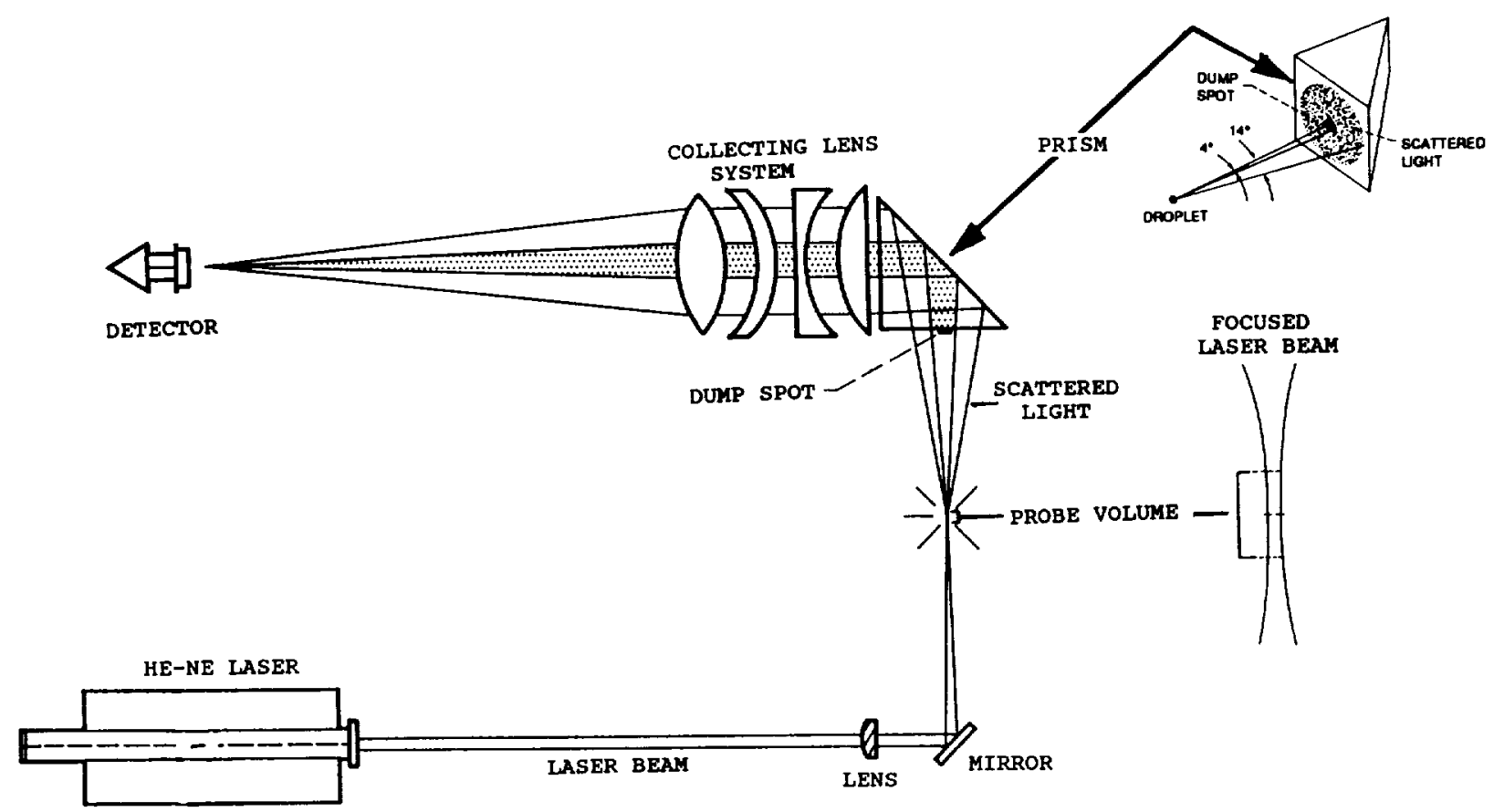

FIG. 1. Optical configuration of the FSSP. 
of the FSSP while measuring the output voltage from the detector after it has been filtered and amplified. This measurement relates the measured voltage to droplet size and accounts for laser beam intensity, detector efficiency, and electronic gain. Since the relationship between droplet size and voltage is nonlinear, a theoretical calibration curve is needed to complete the calibration over the entire range. If there are errors in the curve then the calibration will only be correct at the diameter droplet used in the calibration, and sizing errors can increase at larger and smaller diameters.

Since water droplets are available at only a limited number of diameters, substitute particles can be used to generate more points on the calibration curve. Most popular are glass beads of a known diameter. This requires calculation of a calibration curve for glass beads as well as one for water droplets. The relationship between glass-bead diameter and water-droplet diameter is determined by identifying the diameters of the water droplets that scatter the same power as a given diameter glass bead. Then for any given diameter glass bead there exists an equivalent-size water droplet that scatters the same amount of light. Calibration is then carried out using sets of beads in many diameters over the range of the instrument. If the glass beads are available at only a few diameters then the gaps in the calibration must be filled in using the computed calibration curve. The accuracy of the calibration curve becomes important if glass beads at only a few diameters are used.

\section{Calibration curve equations and enhancements to Mie theory}

The equation that is used to calculate the calibration curve for the FSSP is

$$
\begin{array}{r}
P(d)=I_{0} \frac{1}{k^{2}} \int_{0}^{2 \pi} \int_{\theta_{0}}^{\theta_{1}}\left[\frac{\left|S_{1}(\theta, \phi)\right|^{2}+\left|S_{2}(\theta, \phi)\right|^{2}}{2}\right] \\
\times \sin (\theta) d \theta d \phi,
\end{array}
$$

where $P$ is the power of the scattered light collected over the angles $\theta_{0}$ and $\theta_{1}$ (typically $4^{\circ}-14^{\circ}$ ), $d$ is the diameter of the particle, $k$ is $2 \pi / \lambda, \lambda$ is the wavelength of the unpolarized laser light $(0.6328 \mu \mathrm{m})$, and $I_{0}$ is the intensity of the incident laser beam. The angles $\theta$ and $\phi$ are the scattering angles from the particle as defined by spherical coordinates. The quantities $S_{1}$ and $S_{2}$ are the complex amplitude functions that represent the transverse electric and transverse magnetic field polarizations (van de Hulst 1981). These are calculated using the far-field Mie equations (Wiscombe 1980) and need as input the wavelength of the laser beam, diameter of the particle, and index of refraction of the particle. In practice Eq. (1) is numerically integrated and all of the constants are grouped together:

$$
P(d)=C \int_{\theta_{0}}^{\theta_{1}}\left[\left|S_{1}(\theta)\right|^{2}+\left|S_{2}(\theta)\right|^{2}\right] \sin (\theta) d \theta \text {. }
$$

Note that the integral over $\phi$ is also a constant and it is grouped with the other constants as well.

The inputs to the standard Mie equations do not contain any details about the diameter of the laser beam, its intensity profile, or its divergence. In fact, an assumption for standard Mie theory is that the particle scattering the light is uniformly illuminated by a plane wave that has an infinite extent and is of constant intensity everywhere.

These rather restricting assumptions about the illumination are no longer necessary because of recent enhancements to Mie theory. In the early 1980 s scattering by a Gaussian beam was solved using a plane wave expansion of the incident focused beam (Yeh et al. 1982). This method has certain computational difficulties and as a result other solutions to the problem were pursued. One solution made use of the Davis firstorder Gaussian beam approximation (Davis 1979). First the problem of scattering from a spherical particle centered in a Gaussian beam was solved (Gouesbet et al. 1985). Later the same problem was solved using the localized approximation, which speeded up the calculation considerably (Grehan et al. 1986; Maheu et al. 1987). Eventually the problem of scattering from a spherical particle at an arbitrary position in a Gaussian beam was solved; however, the calculation involved time-consuming three-dimensional numerical integrations (Gouesbet et al. 1988) or a somewhat faster twodimensional surface integral (Barton et al. 1988). In 1990 the localized approximation was determined for this problem as well (Gouesbet et al. 1990), which enabled these scattering calculations to be performed on a microcomputer in a reasonable amount of time. The equations using the localized approximation and computational considerations are given in Lock (1993).

Although the Gaussian model of a laser beam is useful for a wide variety of applications, it is not appropriate for the FSSP because the beam in the FSSP is multimode rather than a Gaussian shape. As shown in Fig. 2 the intensity profile across the focal plane of an FSSP is quite irregular. However, the principle of superposition does enable us to model an irregularly shaped laser beam like the FSSP's as a superposition of Gaussian beams, within certain limitations. A theoretical limitation utilizing the Gaussian superposition involves the width of the Gaussian beams. Since the Gaussian beam formalism is based on the Davis model, which is essentially a truncated Taylor series expansion, the width of beam cannot be made arbitrarily small. For the Davis model and a He-Ne laser wavelength, the minimum half-width is approximately $15 \mu \mathrm{m}$. There is also a practical limitation of using the Gaussian superposition technique. The run time of the computer code can become prohibitive if a large number of Gaussian beams need to be superposed to create some arbitrary beam shape. All the calculations presented in this paper were performed on a $33-\mathrm{MHz} 80386$ micro- 
computer with a superposition of 19 Gaussian beams. Calculation of calibration curves with droplet diameters from 1 to $100 \mu \mathrm{m}$ in diameter took several hours of run time.

Knowing the general profile and diameter of the FSSP's laser beam enabled us to simulate one with 19 Gaussian beams as shown in Fig. 3. The simulated FSSP beam was constructed so that it had roughly the same features as the actual beam: sharp intensity decrease at the edges, similar diameter, four or five intensity fluctuations across the top of the beam, and the intensity fluctuations are of a similar magnitude as in the actual beam. The equations describing the simulated beam in the probe volume are:

$$
\begin{array}{r}
I(x, y)=\left\{\sum_{i=1}^{n} \exp \left[-\frac{\left(x-x_{0_{i}}\right)^{2}}{w^{2}}\right]\right. \\
\left.\quad \times \exp \left[-\frac{\left(y-y_{0_{i}}\right)^{2}}{w^{2}}\right]\right\}^{2}
\end{array}
$$

$$
\begin{aligned}
& x_{0_{i}}=0, \\
& y_{0_{i}}=0, \\
& x_{0_{i}}=A \cos \left[(i-2) \frac{\pi}{3}\right], \\
& y_{0_{i}}=A \sin \left[(i-2) \frac{\pi}{3}\right], \quad 2 \leqslant i \leqslant 7 \\
& x_{c_{i}}=2 A \cos \left[(i-8) \frac{\pi}{6}\right], \\
& y_{0_{i}}=2 A \sin \left[(i-8) \frac{\pi}{6}\right], \quad 8 \leqslant i \leqslant 19,
\end{aligned}
$$

where $n=19$ is the number of Gaussian beams, $A$ $=46 \mu \mathrm{m}$ is the beam spacing, and $w=23 \mu \mathrm{m}$ is the Gaussian beams' half-width.

\section{Modified calibration curves and FSSP response functions}

Using the equations for the Davis first-order Gaussian laser beam [Eq. (49) from Lock for $S_{1}$ and $S_{2}$ substituted into Eq. (2)] and the principle of superposition (which together will be referred to as the new Mie formalism), we recalculated the water-droplet and glassbead calibration curves and compared them with the calibration curves calculated with standard plane-wave Mie theory. Also as a check, we calculated the calibration curves using a single, very wide Gaussian beam ( $w=500 \mu \mathrm{m}$ ) and got excellent agreement with standard Mie theory. In the limit as $w \rightarrow \infty$ the Gaussian beam transforms into a plane wave.

Figures 4 and 5 show the water-droplet and glassbead calibration curves. For these plots the index of refraction was taken to be $m=1.333$ for water and $m$

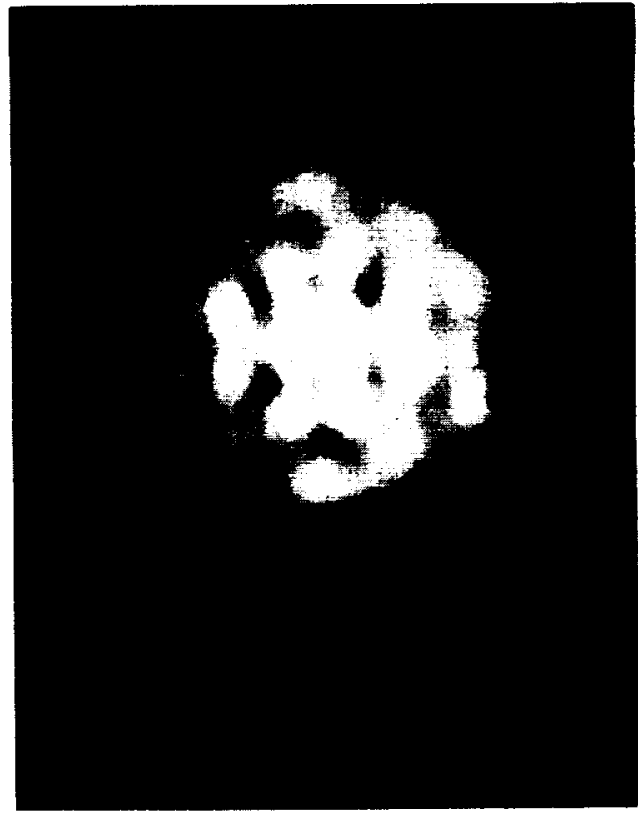

Fig. 2. Photograph of the FSSP laser beam profile at the focal plane.

$=1.51$ for glass. Additionally, the optical collection angles were assumed to be $4^{\circ}-14^{\circ}$ and the beam diameter was $230 \mu \mathrm{m}$ with the profile shown in Fig. 3. Also, the particle was assumed to be centered in the laser beam.

There are several interesting features to note about the calibration curves in Figs. 4 and 5. First, the curves that were generated using standard Mie theory agree quite well with the new formalism for small particles $(<10 \mu \mathrm{m})$. This is because a smaller particle centered in the FSSP beam will be uniformly illuminated, which is one of the assumptions for standard Mie theory. Also note in Fig. 4 (see inset) that if the instrument output were interpreted using standard Mie theory, then a 50$\mu \mathrm{m}$ droplet will be incorrectly sized at $45.5 \mu \mathrm{m}$. Similar errors exist for the glass-bead curve in Fig. 5 as well. This undersizing of larger-size glass beads had been previously commented on by Dye and Baumgardner (1984) but they didn't account for the reason. However, it turns out that this error is compensated for when water-droplet diameter is related to glass-bead diameter. This can be seen in Fig. 6, which compares the glass-bead diameter to an equivalent water-droplet diameter. This relationship will be referred to as the transfer function. The transfer functions in Fig. 6 were calculated using both standard Mie theory and the new formalism for comparison. As can be seen in the figure, the curves are nearly indistinguishable.

One may be tempted to conclude from the similarities of the two curves in Fig. 6 that the more accurate model of the FSSP is not necessary for a more accurate calibration. Although the relationship between glass- 


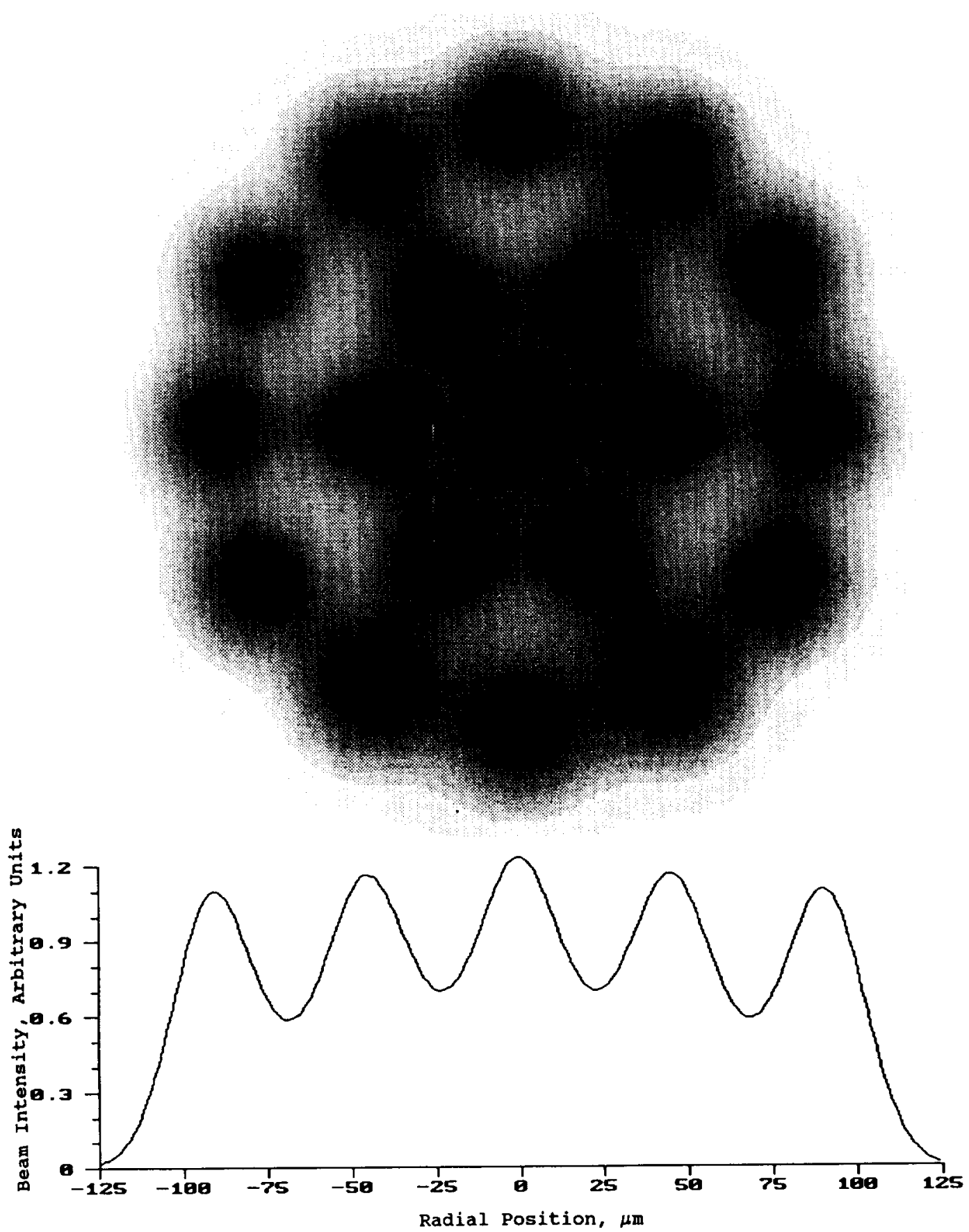

FIG. 3. Simulated FSSP laser beam constructed from a superposition of 19 Gaussian beams.

bead diameter and equivalent water-droplet diameter (the transfer function) is linear and not sensitive to the details of the scattering model, the relationship between water-droplet diameter and instrument response (the calibration curve) is highly nonlinear and is dependent on the details of the scattering model. It is the calibration curve that contains the information needed to determine the details of the FSSP's calibration such as voltage thresholds, which determine the size bin boundaries.

The new formalism is also useful for calculating the FSSP response as droplets of different diameters traverse the probe volume. Figures $7 \mathrm{a}-\mathrm{d}$ show the scattered power as a water droplet passes through the center of the probe volume. For the case of a $10-\mu \mathrm{m}$ water droplet (Fig. 7a), its diameter is smaller than the os- 


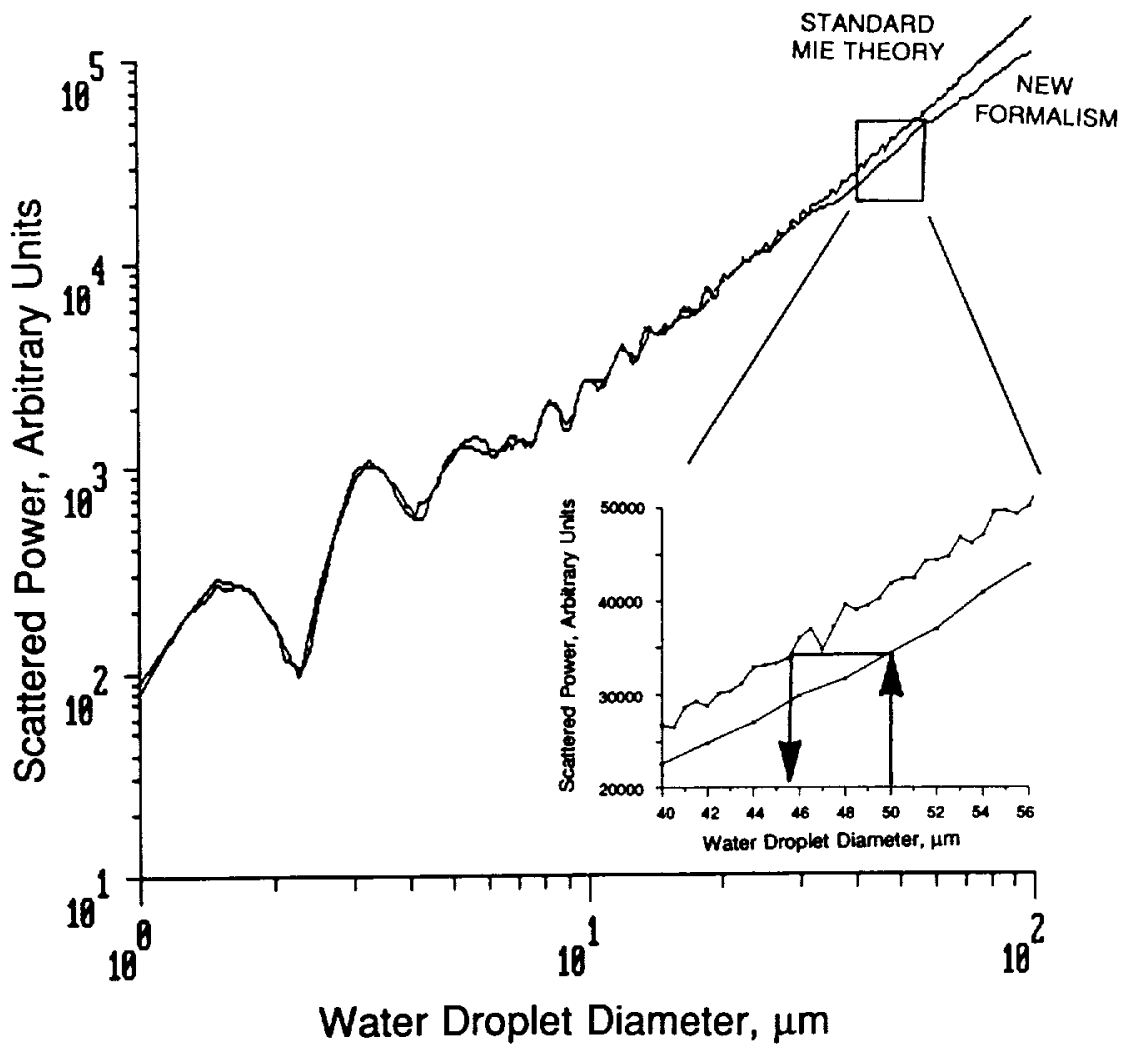

FIG. 4. Water-droplet calibration curves.

cillations in the laser beam profile, and the scattered power curve traces out the beam profile. Larger droplets span several oscillations in the beam profile, and the scattered power represents an average over those oscillations. For $100-\mu \mathrm{m}$ droplets (Fig. 7d), all details about the beam are lost. These plots are similar to those given in Hovenac (1989) for different diameter pinholes passing through the FSSP.

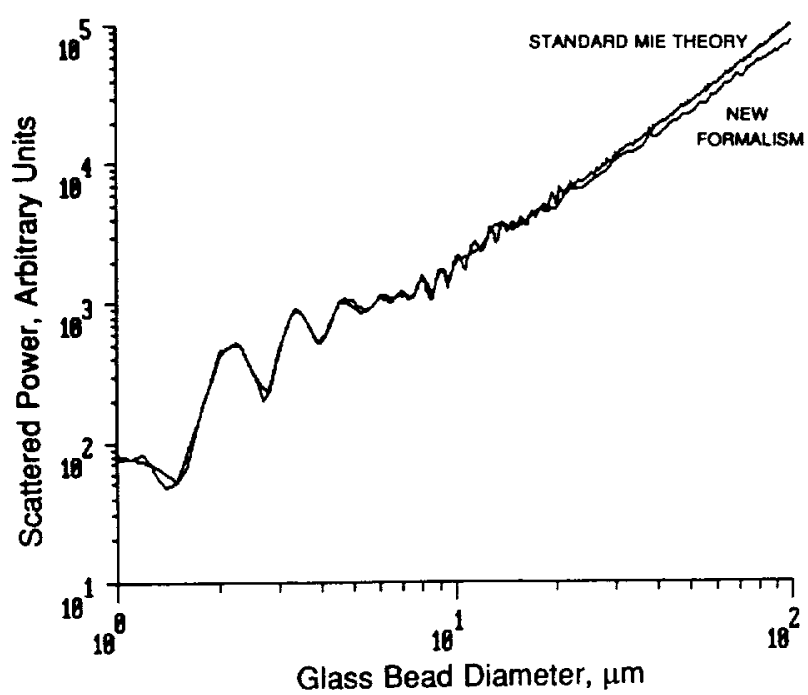

FIG. S. Glass-bead calibration curves.
The new formalism can be used to calculate FSSP response functions. Response functions are similar to the calibration curves except they are calculated in a manner closer to the way the FSSP makes the size determination. Scattering is calculated for a particle at a series of 21 discrete locations in the beam as if the particle were traversing the FSSP laser beam. The maximum scattering is determined from the 21 cal-

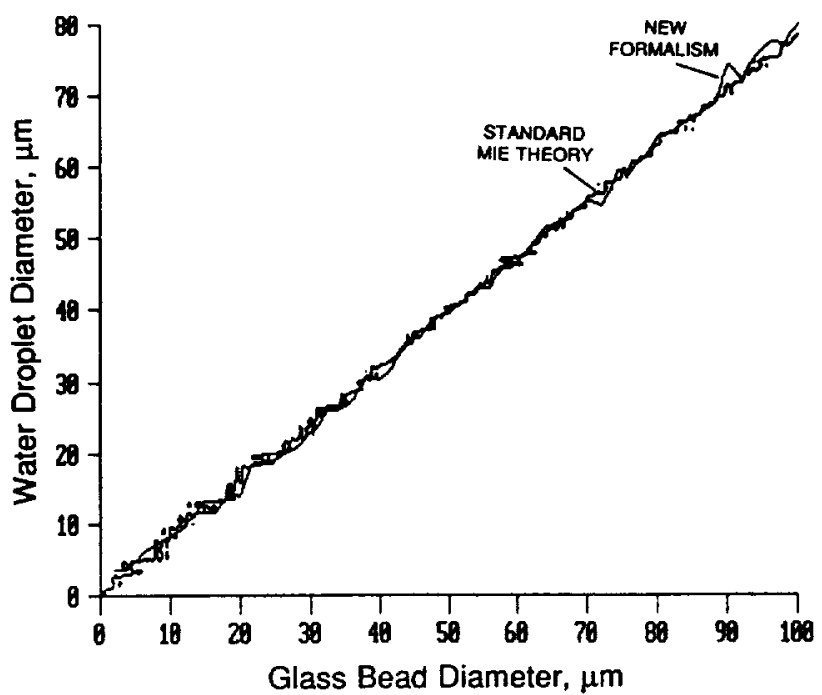

FIG. 6. Glass-bead to water-droplet transfer function. 
Scattering From a 10 in Dianeter Droplet
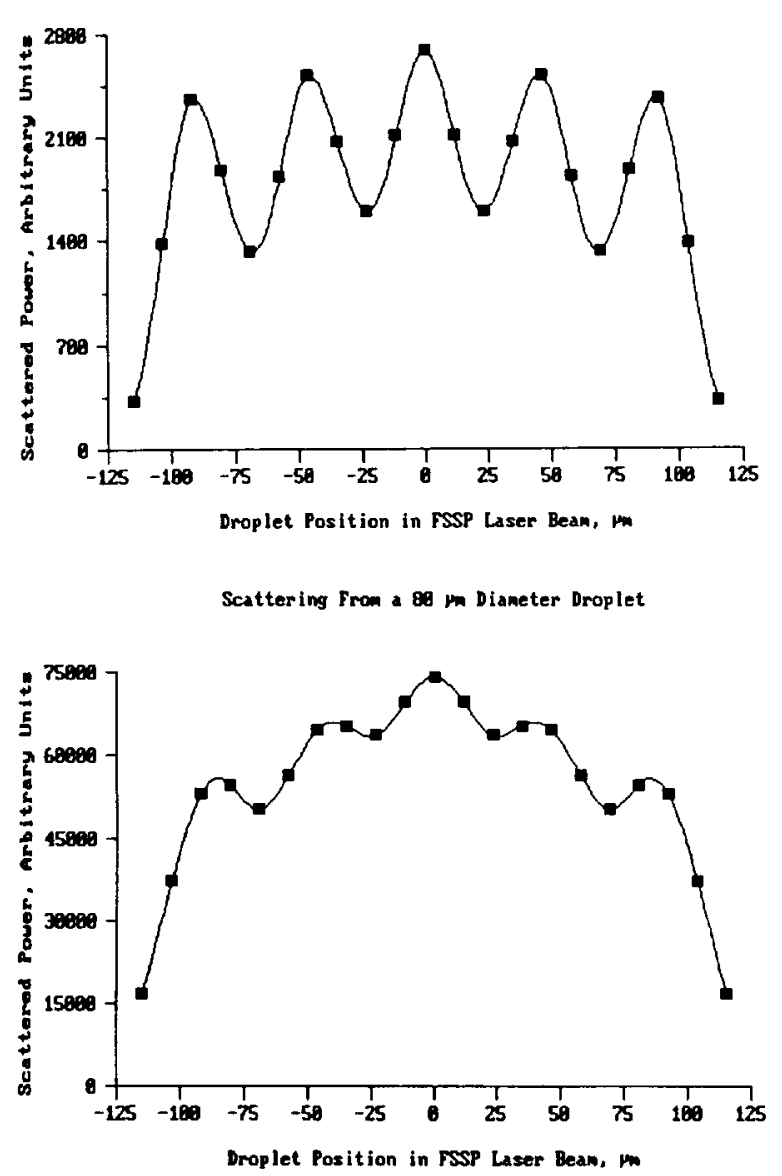

Scattering From a 50 mianeter Droplet

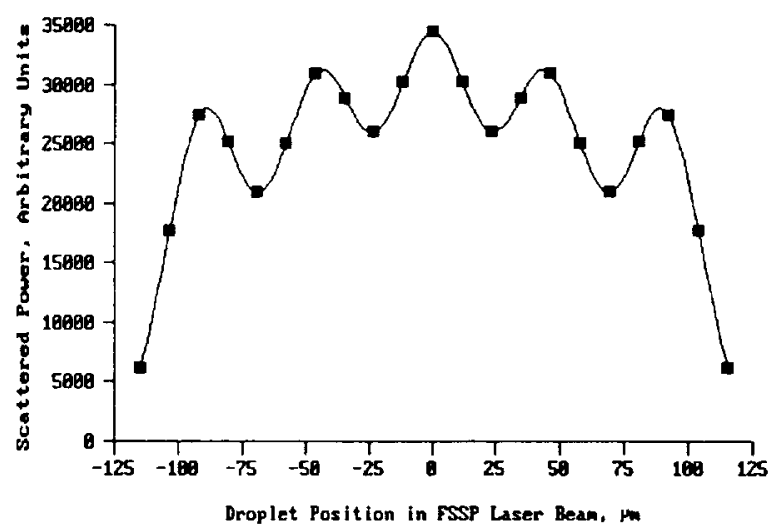

Scattering From a 160 in Dianeter Droplet

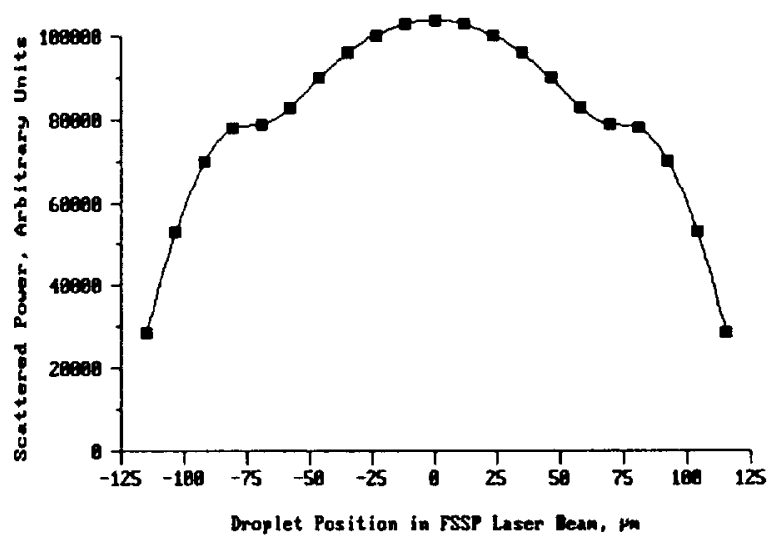

FIG. 7. Calculated FSSP response to different diameter water droplets going through the center chord of the FSSP probe volume. Filled squares indicate 21 locations where the calculation was performed.

culations in the assumed particle path just as the FSSP would determine the maximum scattering for a real particle traversing a continuous path through the beam. Also, four different paths or trajectories of particles through the laser beam were assumed: 1) the particle traversed the center of the depth of field and the radial center of the beam, 2) the particle traversed a chord through the beam not at the radial center but in the center of the depth of field, 3 ) the particle traversed the beam $1 \mathrm{~mm}$ from the center of the depth of field and through the radial center, 4) the particle traversed a chord through the beam not at the radial center and $1 \mathrm{~mm}$ from the center of the depth of field. The chords, which were assumed to be away from the radial center of the beam, had lengths of $\pi / 4$ beam diameters ( 181 $\mu \mathrm{m})$. This chord was chosen because it represents the maximum distance a particle can be away from the radial center of the beam and still pass the FSSP transit time criterion (Baumgardner et al. 1985) and thus be accepted for sizing.

Figure 8 shows the response functions for water droplets going through the varied trajectories as described above. This shows the theoretical uncertainty of sizing water droplets in the FSSP. For example, assume the FSSP is calibrated using the new calibration curve of Fig. 4. A size bin on the FSSP that measures

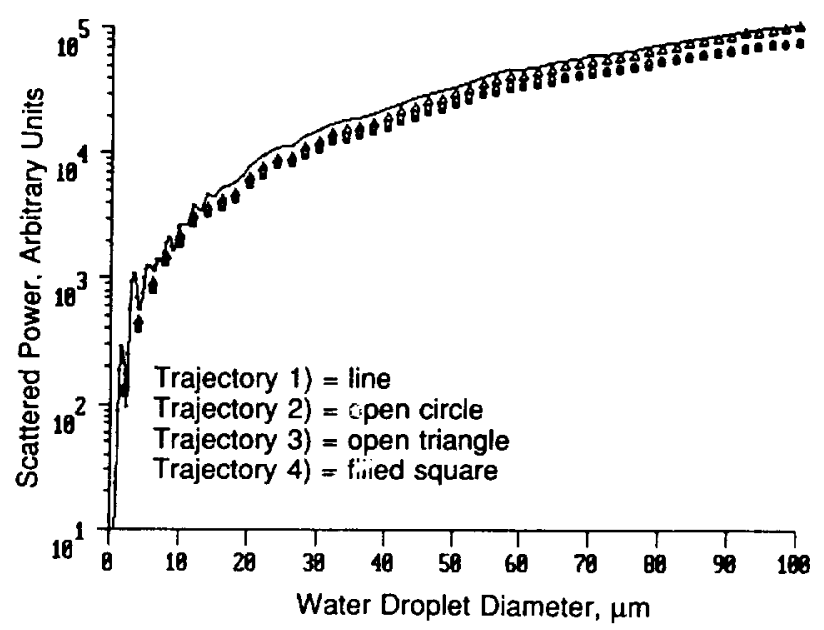

FIG. 8. FSSP response function for four trajectories of water droplets through the probe volume of the FSSP. 


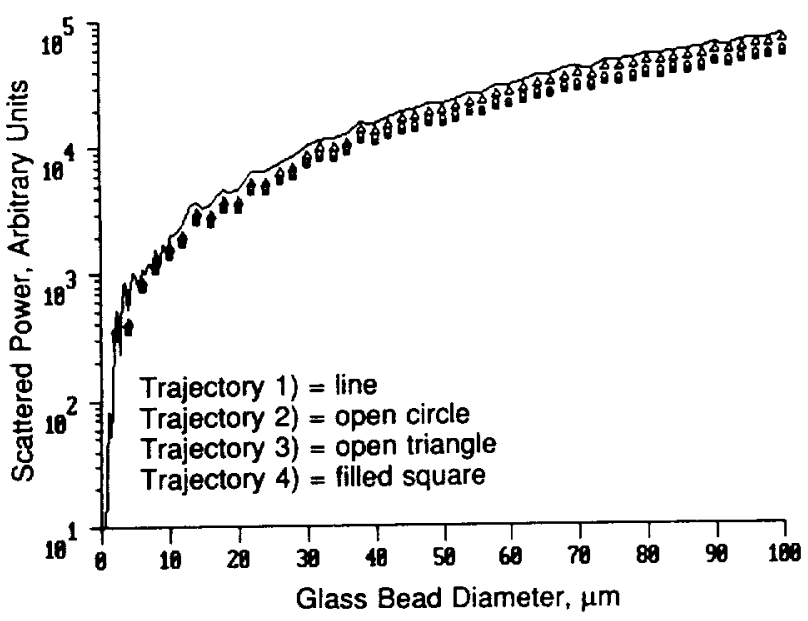

FIG. 9. FSSP response function for four trajectories of glass beads through the probe volume of the FSSP.

droplets between 44 and $47 \mu \mathrm{m}$ corresponds to droplets scattering light between $2.70 \times 10^{4}$ and $3.07 \times 10^{4}$ units. However, larger droplets crossing the laser beam at other trajectories can also scatter light in this same power range. For example, a $55-\mu \mathrm{m}$ droplet that traverses a path as defined by condition 4 above will scatter light at $3.00 \times 10^{4}$ units, which is within the 44-47$\mu \mathrm{m}$ bin. It should be noted that the probability is small for a $55-\mu \mathrm{m}$ droplet to be sized in the $44-47-\mu \mathrm{m}$ size bin because of all the possible trajectories that a 55$\mu \mathrm{m}$ droplet can take through the probe volume; it must take the trajectory that goes through the edge of the depth of field and through the edge chord of the probe volume. Similar errors can occur for smaller-diameter droplets as well. For example, a $16-\mu \mathrm{m}$ droplet that traverses a path as defined by condition 4 will scatter the same amount of light as a $11.7-\mu \mathrm{m}$ droplet that traverses a path as defined by condition 1 .

Figure 9 shows the response function for glass beads. As can be seen, glass beads are also subject to the same sizing errors as water droplets. This effect has been measured by Hovenac (1989) using rotating pinholes going through random trajectories of the probe volume and is also apparent whenever monodisperse glass beads are blown through the FSSP during calibration.

\section{Conclusions}

Scattering calculations using a more detailed model of the FSSP laser beam were carried out. From this model, new calibration curves and response functions were calculated for an FSSP with a laser beam $230 \mu \mathrm{m}$ in diameter and optical collection angles of $4^{\circ}-14^{\circ}$. The difference between calibration curves calculated using standard Mie theory versus calibration curves calculated using the new formalism is small for droplet diameters less than $10 \mu \mathrm{m}$ but the difference increases with diameter. At droplet diameters of $50 \mu \mathrm{m}$ the difference increases to about $10 \%$. When using glass beads to calibrate the FSSP, calibration errors can be minimized, by using glass beads of many different diameters, over the entire range of the FSSP. If the FSSP is calibrated using one-diameter glass beads, then the new formalism is necessary to extrapolate the calibration over the entire range.

The response of the FSSP to droplets going through varied trajectories of the beam was also calculated. This calculation showed that droplets as large as $55 \mu \mathrm{m}$ can be sized in the instrument's $44-47-\mu \mathrm{m}$ size bin. This effect is responsible for artificial broadening of the measured droplet size distribution.

\section{REFERENCES}

Barton, J. P., D. R. Alexander, and S. A. Schaub, 1988: Internal and near-surface electromagnetic fields for a spherical particle irradiated by a focused laser beam. J. Appl. Phys., 64, 1632-1639.

Baumgardner, D., and M. Spowart, 1990: Evaluation of the forward scattering spectrometer probe. Part IIl: Time response and laser inhomogeneity limitations. J. Atmos. Ocean. Technol., 7, 666672.

- W. Strapp, and J. E. Dye, 1985: Evaluation of the forward scattering spectrometer probe. Part II: Corrections for coincidence and dead-time losses. J. Atmos. Oceanic Technol., 2, 626632.

Davis, L. W., 1979: Theory of electromagnetic beams. Phys. Rev., A19, 1177-1179.

Dye, J. E., and D. Baumgardner, 1984: Evaluation of the forward scattering spectrometer probe. Part I: Electronic and optical studies. J. Atmos. Oceanic Technol., 1, 329-344.

Gouesbet, G., G. Grehan, and B. Maheu, 1985: Scattering of a Gaussian beam by a Mie scatter center using a Bromwich formalism. J. Opt., 16, 83-93.

,-- and -1988 : Computations of the $g_{n}$ coefficients in the generalized Lorenz-Mie theory using three different methods. Appl. Opt., 27, 4874-4883.

,+- and $-1990:$ Localized interpretation to compute all the coefficients $g_{n}^{m}$ in the generalized Lorenz-Mie theory. $J$. Opt. Soc. Am., A7, 998-1007.

Grehan, G., B. Maheu, and G. Gouesbet, 1986: Scattering of laser beams by Mie scatter centers: Numerical results using a localized approximation. Appl. Opt., 25, 3539-3548.

Hovenac, E. A., 1989: Droplet sizing instruments used for icing research: Operation, calibration, and accuracy. NASA CR 182293 DOT/FAA/CT-89/13, $54 \mathrm{pp}$.

- and E. D. Hirleman, 1991: Use of rotating pinholes and reticles for calibration of cloud droplet instrumentation. J. Atmos. Oceanic Technol., 8, 166-171.

Lock, J. A., 1993: The contribution of high-order rainbows to the scattering of a Gaussian laser beam by a spherical particle. $J$. Opt. Soc. Am. A 10, 693-706.

Maheu, B., G. Grehan, and G. Gouesbet, 1987: Generalized LorenzMie theory: First exact values and comparisons with the localized approximation. Appl. Opt., 26, 23-25.

Pinnick, R. G., D. M. Garvey, and L. D. Duncan, 1981: Calibration of Knollenberg FSSP light-scattering counters for measurement of cloud droplets. J. Appl. Meteor., 20, 1049-1057.

van de Hulst, H. C., 1981: Light Scattering by Small Particles. Dover, $470 \mathrm{pp}$.

Wiscombe, W. J., 1980: Improved Mie scattering algorithms. Appl. Opt., 19, 1505-1509.

Yeh, C., S. Colak, and P. Barber, 1982: Scattering of sharply focused beams by arbitrarily shaped dielectric particles: An exact solution. Appl. Opt., 21, 4426-4433. 